\title{
A fractional multistep method for solving a class of linear fractional differential equations under uncertainty
}

\begin{abstract}
The objective of this research has been devoted to solve linear fuzzy fractional differential equations (FFDEs) of the Caputo sense. The basic idea is to develop a fractional linear multistep method for solving linear FFDEs under fuzzy fractional generalized differentiability. For safely illustrating the advantages and potential of the presented method, a comparison with the fractional Euler method has to be analyzed in depth. We are interested in using a simple method to obtain gripping results.
\end{abstract}

Keyword: Fuzzy fractional differential equations (FFDEs); Fractional linear multistep method (FLMM) 